\title{
Sensorimotor Prediction and Memory in Object Manipulation
}

\author{
J. RANDALL FLANAGAN, SARA KING, Queen's University \\ DANIEL M. WOLPERT, Institute of Neurology, University College London, \\ and ROLAND S. JOHANSSON, Umeå University
}

\begin{abstract}
When people lift objects of different size but equal weight, they initially employ too much force for the large object and too little force for the small object. However, over repeated lifts of the two objects, they learn to suppress the size-weight association used to estimate force requirements and appropriately scale their lifting forces to the true and equal weights of the objects. Thus, sensorimotor memory from previous lifts comes to dominate visual size information in terms of force prediction. Here we ask whether this sensorimotor memory is transient, preserved only long enough to perform the task, or more stable. After completing an initial lift series in which they lifted equally weighted large and small objects in alternation, participants then repeated the lift series after delays of 15 minutes or 24 hours. In both cases, participants retained information about the weights of the objects and used this information to predict the appropriate fingertip forces. This preserved sensorimotor memory suggests that participants acquired internal models of the size-weight stimuli that could be used for later prediction.
\end{abstract}

Résumé Lorsque les gens soulèvent des objets de tailles différentes mais de poids égal, ils déploient au départ trop de force dans le cas du gros objet et trop peu dans celui du petit. Cependant, après avoir soulevé à plusieurs reprises les deux objets, ils apprennent à mettre de côté l'association taille-poids employée jusque-là pour évaluer l'effort nécessaire, et ajustent adéquatement la force à appliquer au poids effectivement égal des objets. Ainsi, la mémoire sensori-motrice des levées précédentes vient supplanter l'information visuelle sur la taille de l'objet pour ce qui est de prédire la force requise. Nous nous demandons donc si cette mémoire sensori-motrice est transitoire, c'est-à-dire conservée seulement le temps d'effectuer la tâche, ou si elle subsiste davantage. Après une première série de levées pendant laquelle les sujets soulevaient en alternance des objets de grande et de petite taille, mais de poids égal, ils ont repris la même série de levées 15 minutes ou 24 heures plus tard. Dans les deux cas, les sujets ont retenu l'information relative au poids des objets et l'ont utilisée pour prédire correctement la force à appliquer avec le bout des doigts. Le fait que cette mémoire sensori-motrice subsiste suggère que les sujets acquièrent des modèles internes quant aux stimuli taille-poids qui peuvent être appliqués à des prédictions ultérieures.

Most of us will recall having fallen victim to a mischievous older sibling, cousin, or dubious friend who passed us an empty box while pretending it was very heavy. When we took the box, and the bait, our arms flailed upwards. This trick demonstrates that when we interact with objects, we anticipate the forces required to accomplish the task at hand. Although it may occasionally result in large movement errors, predictive or anticipatory control is essential for skilled object manipulation, whether wielding a tennis racquet or simply picking up a cup. The alternative control strategy - reactive control based on sensory feedback - becomes critical when predictions are erroneous or unavailable. However, because of large time delays associated with receptor transduction, neural conduction and processing, and muscle contraction, reactive control cannot support the fast and accurate movements observed in most natural actions.

To learn and maintain the ability to generate motor commands required for desired actions, the motor system must also predict the sensory consequences of these motor commands. Thus, skilled actions, such as those involved in object manipulation, entail both motor command estimation and sensory prediction. The ability to predict the sensory consequences of motor commands may be based on internal forward models that mimic the behaviour of motor system and manipulated objects (Flanagan \& Wing, 1997; Jordan \& 
Rumelhart, 1992; Miall \& Wolpert, 1996; Wolpert, Ghahramani, \& Jordan, 1995). The idea is that the brain generates a sensory prediction (corollary discharge; Sperry, 1950) by sending a copy of the motor commands (efference copy; Von Holst, 1954) to the forward model. Conversely, the ability to estimate the motor commands required to achieve a desired action may be based on internal inverse models that translate from desired outcomes to motor commands (Kawato, 1999; Kawato, Furukawa, \& Suzuki, 1987; Shadmehr \& Mussa-Ivaldi, 1994). These two facets of predictive control - sensory and motor prediction - have been investigated vigorously in the context of precision lifting (see Johansson, 1998 for a review). In the current paper, we will focus on motor and sensory predictions related to weights of objects to be lifted.

\section{FORCE COORDINATION IN PRECISION LIFTING}

When lifting small objects, people typically place the tips of the index finger and thumb on either side of the object in what is referred to as a precision grip. To lift the object, a vertical load force greater than the weight of the object must be applied. Importantly, for a given height and speed of lifting, the rate at which individuals increase load force is scaled to the expected weight of the object; the greater the expected weight of the object, the more rapid the increase in load force (Johansson \& Westling, 1988). This rate scaling is observed as soon as load force starts to increase and well before lift-off when sensory confirmation of weight is available. Therefore, it is clearly anticipatory or predictive. The peak rate of change of load force therefore provides an index of expected weight. When lifting objects with a precision grip, horizontal grip forces are increased in parallel with load force and prevent the object from slipping. The rate of change of grip force, also observed early in the lift, provides an additional index of expected weight but also depends on the friction between the object and fingertips and the individual's safety margin against slips. If the prediction of object weight is erroneous, then the object will either lift off sooner than expected or may still not have lifted off at the expected time. Either of these unexpected events triggers a reflex-mediated change in force output within about $100 \mathrm{~ms}$ (Johansson \& Westling, 1988; Westling \& Johansson, 1987). Thus, the sensorimotor system reacts quickly to both the presence of an unexpected sensory event and the absence of an expected sensory event (Johansson \& Cole, 1994). The parallel control of grip force and load force observed in precision lifting is also observed when moving hand-held objects during which accelerationdependent load forces are experienced (Flanagan \& Wing, 1993, 1995; Flanagan \& Tresilian, 1994).
FACTORS THAT INFLUENCE SENSORIMOTOR PREDICTION A number of factors influence the anticipatory scaling of fingertip forces in precision lifting. These include visual and haptic information about object size (Gordon, Forssberg, Johansson, \& Westling, 1991a-c) and shape (Jenmalm, Dahlstedt, \& Johansson, 2000; Jenmalm, Goodwin, \& Johansson, 1998; Jenmalm \& Johansson, 1997), visual information about the weight distribution of the object (Goodwin, Jenmalm, \& Johansson, 1998; Jenmalm et al., 2000; Johansson, Backlin, \& Burstedt, 1999; Wing \& Lederman, 1998), and object identity (Gordon, Westling, Cole, \& Johansson, 1993). Thus, anticipatory force control is clearly influenced by the geometric properties of the object to be lifted. However, immediate sensorimotor memory obtained from previous lifts is also a powerful factor influencing predictive force control. When the weight of a repeatedly lifted object is unexpectedly changed (without changing the visual appearance of the object), individuals generate inappropriate forces on the first lift. However, quite accurate force scaling is observed by the second lift (Johansson \& Westling, 1988). For example, when lifting everyday objects that have variable density (e.g., a milk box that may be empty or full), people only perform erroneously once. Similar one-trial adaptation is observed following unexpected changes in surface friction (Johansson \& Westling, 1984), object shape (Jenmalm \& Johansson, 1997; Jenmalm et al., 1998, 2000), and mass distribution (Johansson et al., 1999). This adaptation indicates that people can rapidly update their sensorimotor memory, or internal model, pertaining to the physical properties of the object.

CONFLICTING WEIGHT CUES AND THE SIZE-WEIGHT ILLUSION

Given that multiple sources of information can influence force prediction, one can ask about their relative importance. In a recent paper, the relative contributions of sensorimotor memory and visual size cues to fingertip force prediction was investigated within the context of the size-weight illusion (Flanagan \& Beltzner, 2000). This illusion, first documented over 100 years ago (Charpentier, 1899; Murray, Ellis, Bandomir, \& Ross, 1999), refers to the fact that people judge the smaller of two equally weighted objects to be heavier. According to a leading theory, the size-weight illusion arises from a mismatch between expected sensory feedback and actual sensory feedback (Davis \& Roberts, 1976; Granit, 1972; Ross, 1969). The idea is that the smaller object is judged to be heavier because it is heavier than expected. That is, the actual sensory information obtained during the lift differs from the expected sensory infor- 
mation estimated from the size of the object.

To test this sensory-mismatch theory, participants were asked to repeatedly lift two cubes of different size but equal weight in alternation (Flanagan \& Beltzner, 2000). As expected, participants were initially fooled by the visual size of the cubes and generated too much force when lifting the large cube (which had an uncommonly low density) and too little force when lifting the small cube (which had an uncommonly high density). However, after lifting each object 5 to 10 times, participants learned to scale their fingertip forces appropriately to the true (equal) weights of the cubes. That is, not only did they employ the same forces when lifting the two cubes, they also scaled these forces such that the force was neither too large nor too small. This indicates that their motor systems expected the two objects to weigh the same. That is, the motor system learned to suppress the size-weight association when controlling force. Nevertheless, all participants still experienced the size-weight illusion and the strength of the illusion was undiminished. Thus, we were able to disprove the sensory-mismatch hypothesis. Our results indicate that the size-weight illusion can be caused by high-level cognitive and perceptual factors (see also Ellis \& Lederman, 1998) and demonstrate that the sensorimotor system can operate independently of the cognitive/perceptual system. Thus, the results provide further support for the notion that sensory information can be processed separately, depending on whether it is used for action or perception (Goodale, Meenan, Bülthoff, Nicolle, Murphy, \& Racicot, 1994; Goodale, Milner, Jakobson, \& Carey, 1991; Milner \& Goodale, 1995).

Although we demonstrated that sensorimotor memory that develops over repeated lifts eventually dominates over visual size cues in terms of predictive control of force, we did not examine the stability of this sensorimotor memory over time. The aim of the present paper was to investigate the persistence of the sensorimotor memory developed when lifting sizeweight stimuli for which size-weight associations are unreliable. One possibility is that this sensorimotor memory is fleeting such that it is persists only for the current series of lifts. However, the memory may also be longer lasting such that information about the weights of the size-weight stimuli can be recalled later and used for accurate prediction. To address this question, we first asked participants to lift a large cube and an equally weighted small cube 20 times each in alternation. After a delay of 15 minutes or 24 hours, the participants completed a second series of alternating lifts.

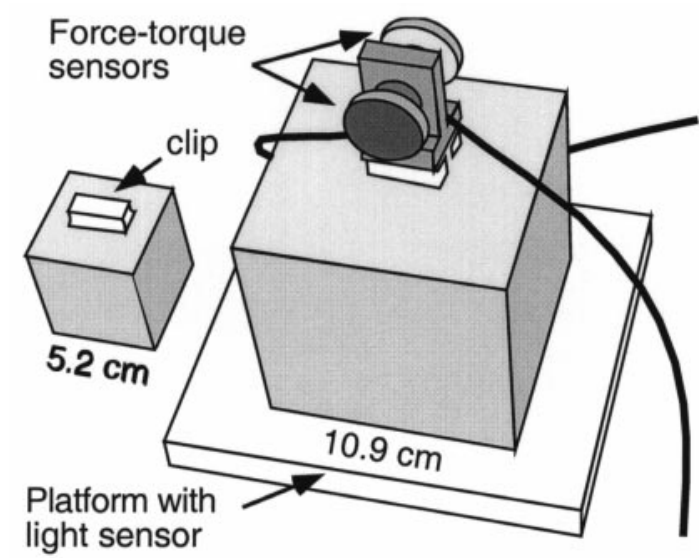

Figure 1. Apparatus and stimuli. Drawing showing the relative sizes of the large and small objects. Participants lifted the objects using a precision grip with the tips of the index finger and thumb on either side of a handle. The handle was attached by clips located on top and in the centre of each object and could be quickly moved from object to object. The handle was instrumented with two sensors that measure the forces and torques applied by each digit. Plastic contact disks $(3 \mathrm{~cm}$ in diameter) were mounted on each sensor and covered in medium grain sandpaper (No. 220). A light-sensitive diode embedded into the centre of the lifting platform recorded object lift-off.

\section{SUBJECTS}

\section{Method}

Twenty-four naïve participants between 18 and 28 years of age participated in this study after providing informed consent. None of the subjects reported neurological or visual impairments.

\section{STIMULI}

The stimuli consisted of two cubes of equal weight but different volume (Figure 1). The volumes of the large and small cubes were $10.9^{3} \mathrm{~cm}^{3}$ and $5.2^{3} \mathrm{~cm}^{3}$, respectively. The cubes were constructed from balsa wood and had similar surface finish and colour. They were weighted with lead-shot, mixed in putty, located in the centres of the cubes.

Participants lifted the cubes by grasping a removable handle mounted on top by a plastic clip (Figure 1). The handle was instrumented with two six-axis force-torque sensors (Nano F/T, ATI Industrial Automation, Garner, NC) that measured the forces and torques applied by the digits in three dimensions. The range and resolution of the sensors are reported elsewhere (Kinoshita, Bäckström, Flanagan, \& Johansson, 1997). The weight of each cube, including the handle, was $3.82 \mathrm{~N}(0.39 \mathrm{~kg})$. The densities of the large $(0.3$ $\mathrm{kg} / \mathrm{l})$ and small $(2.8 \mathrm{~kg} / \mathrm{l})$ boxes straddled the density for most commonly manipulated objects (about $1 \mathrm{~kg} / \mathrm{l}$; Gordon et al., 1993). To record the onset of object liftoff, a light-sensitive diode was embedded into the center of the lifting platform. 


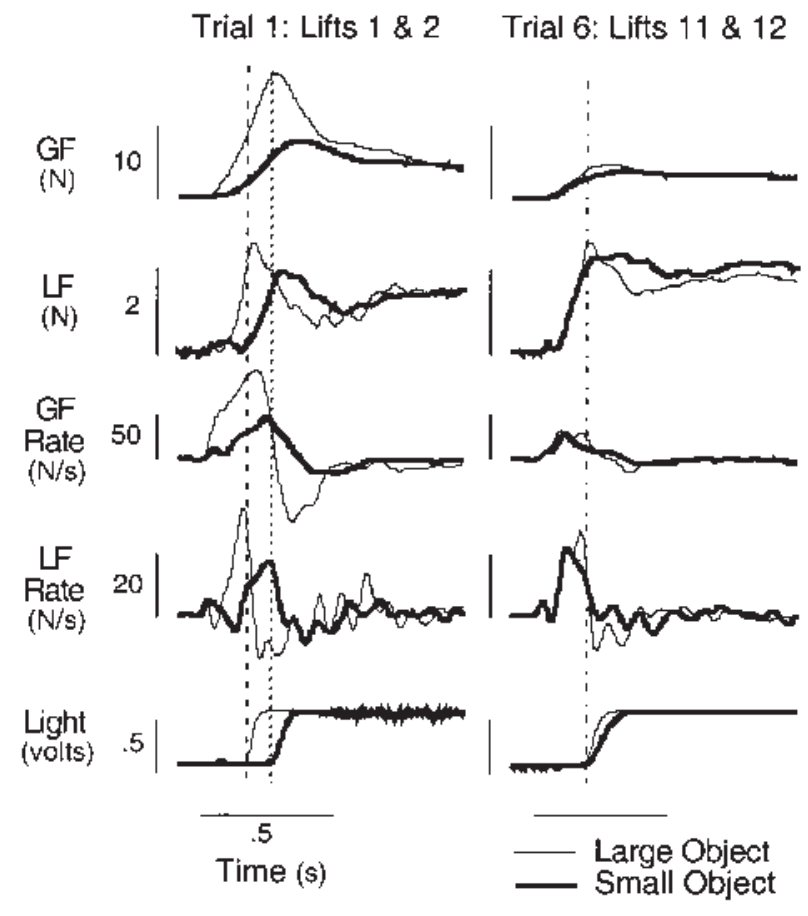

Figure 2. Fingertip force records. Grip force (GF), load force (LF), grip and load force rates, and light sensitive diode recorded in the first trial (lifts 1 and 2; left panel) and the sixth trial (lifts 11 and 12; right panel). This participant lifted the small cube (thick traces) and then the large cube (thin traces) in each trial. In all trials, participants grasped the object and increased grip and load force together until lift-off occurred, signalled by the light diode. In the first trial, grip and load forces were scaled to object size and greater peak force rates were observed for the large cube. By the sixth trial, the forces and force rates were similar for the two objects. The absence of force corrections indicates that the forces were appropriately scaled to object weight. In the first trial, the large cube lifted off earlier (dashed vertical line) than the small object (dotted line) because off the greater load force generated. In the sixth trial, lift-off (dotted-dashed line) occurred at about the same time for both cubes.

\section{PROCEDURE}

Participants grasped the handle with the tips of the thumb and index finger located on the two opposing vertical contact surfaces. On hearing a tone, they were required to grasp and lift the object about $5 \mathrm{~cm}$ about the lifting platform (Figure 1) and then hold it in a stationary position for 3 seconds. Participants were also asked to maintain a constant lifting speed and height. A trial consisted of two lifts, one with the small cube and one with the large cube. The order was counterbalanced across participants. A lift series consisted of 20 trials for a total of 40 lifts. All participants completed an initial lift series. Nine then completed a second lift series 15 minutes later. Fifteen participants completed a second lift series after a delay of 24 hours.
DATA ANALYSIS

Signals from the two force/torque sensors and the light sensitive diode were sampled at $400 \mathrm{~Hz}$. We computed the load force, defined as the resultant force tangential to the grasped surfaces, and the grip force normal to the grasped surfaces. The torques acting in the plane of the contact surfaces and about the normal vector located at the centre of normal force pressure was also computed (see Kinoshita et al., 1997 for details). To obtain grip and load force rates (first time derivative of force), the force signals were smoothed using a fourthorder, zero phase lag, low-pass Butterworth filter (cutoff frequency of $14 \mathrm{~Hz}$ ) and then differentiated using a three-point central difference equation. We recorded forces and torques applied by both the index finger and thumb. However, for simplicity, we only report results pertaining to the index finger. Because participants lifted the objects vertically, very similar results were obtained for both digits. For each trial we determined the peak grip force rate and the peak load force rate. We focused our analysis on peak force rates rather than peak forces because the former occur earlier in the lift and provide a better index of participants' predictions of object weight. In the absence of reactive force corrections, peak force rates are highly correlated with peak forces. However, in the presence of such corrections - triggered by errors in prediction - peak forces can be misleading because they are influenced by reactive control mechanisms. To examine force adaptation, we divided the 20 trials of each lift series into four blocks of five trials. For each block, ANOvA was used to assess the effect of object size (large, small) on peak load force rate and peak grip force rate. The Bonferroni method was used to test post-hoc effects. An alpha level of 0.05 was considered statistically significant.

\section{Results}

ADAPTATION OF FINGERTIP FORCES TO SIZE-WEIGHT STIMULI

All participants initially completed 20 trials (40 lifts) in which they alternately lifted the small and large cubes. To describe the adaptation of fingertip forces over trials, we first present illustrative records from a single participant and then provide data averaged across participants.

Figure 2 shows grip force, load force, and grip and load force rate functions for individual lifts. The light indicating lift-off is also shown. The left panel shows lifts of the small (thick traces) and large (thin traces) cubes from the first trial (lifts 1 and 2, respectively) and the right panel shows lifts of the small and large cubes from the sixth trial (lifts 11 and 12). In the first trial, grip force and load force increased more rapidly when 


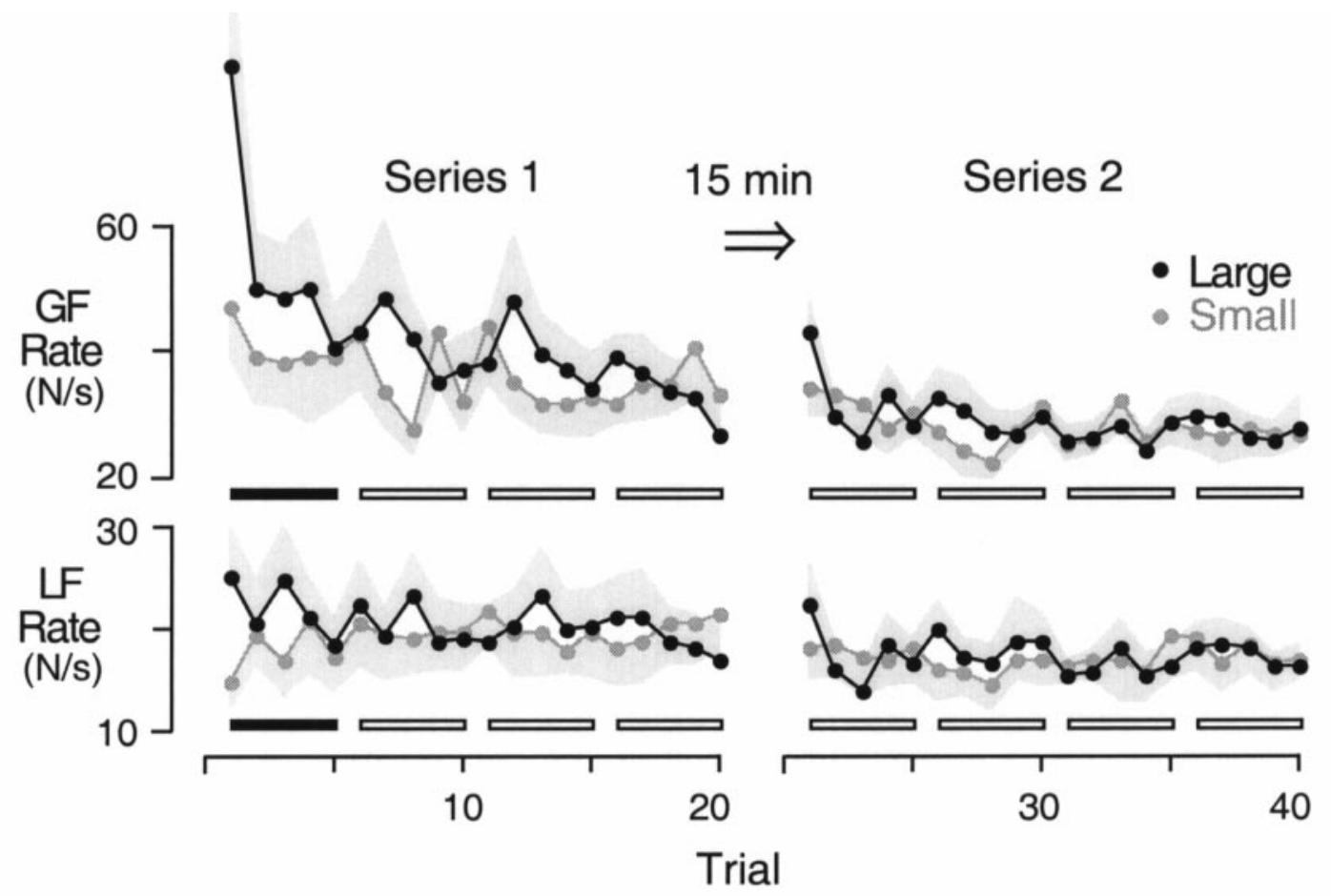

Figure 3. Recall after a 15-minute delay. Peak grip force rate (top) and peak load force rate (bottom) as a function of trial for two lift series separated by 15 minutes. Each black (large cube) and gray (small cube) dot represents an average of nine participants. The gray regions represent one standard error. The horizontal bars indicate, for each block of five trials and for peak load force rate and peak grip force rate separately, whether there was (filled) or was not (open) a significant effect of object size on the peak rate $(p<.05)$.

lifting the large object. This is indicated by the steeper slopes of the grip and load force functions and the larger peak rates of change of grip and load force. As a consequence, the large cube lifted off about $100 \mathrm{~ms}$ early than the small cube. In contrast, in the sixth trial, the force and force rate functions observed for the two cubes were similar prior to lift-off, and lift-off occurred at about the same time. Thus, the participant learned to scale fingertip forces to the true, equal weights of the two cubes. Because sensory information about object weight is not available before lift-off, this accurate scaling of forces indicates that the participant correctly predicted the weights of the cubes.

\section{SENSORIMOTOR MEMORY AFTER A 15-MINUTE DELAY}

Figure 3 shows average peak grip and load force rates for the large and small cubes as a function of trial. Data are shown for two lift series, each consisting of 20 trials, separated by a 15-minute delay. To quantify the history of force adaptation, we divided the trials into four blocks of five trials and compared the peak force rates across the two cubes using ANOva. The horizontal bars in Figure 3 indicate, for both the grip force and load force rates and for each block of trials, whether (filled) or not (open) there was a significant difference between the large and small cubes.
In the first block of five trials, greater peak grip, $F(1,8)=11.06, p=.01$ and load, $F(1,8)=9.75, p=.014$ force rates were observed when lifting the large cube. However, in the subsequent three blocks of five trials in the first lift series, no reliable effects of cube size on either peak grip force rate or peak load force rate were observed $(p>.05$ in all six cases). These results demonstrate that participants initially based their predictions related to object weight on the visual size of the cubes. However, after five trials, they increased grip and load force at similar rates for the two cubes. This finding, which replicates our previous results (Flanagan \& Beltzner, 2000), indicates that, after a period of adaptation, force output was scaled to the true (and equal) weights of the two cubes, independent of their visual size. Thus, participants learned to suppress the mechanisms that use visual size cues to estimate required fingertip forces.

As illustrated in Figure 3, the ratio of peak grip force rate to peak load force rate decreased markedly over the initial few trials in the first lift series. This decrease can be observed for both the large cube (black symbols) and the small cube (gray symbols). The ratio between rates of grip and load force change is influenced by the frictional conditions at the grasped surfaces as well as the grip force safety margin used by 


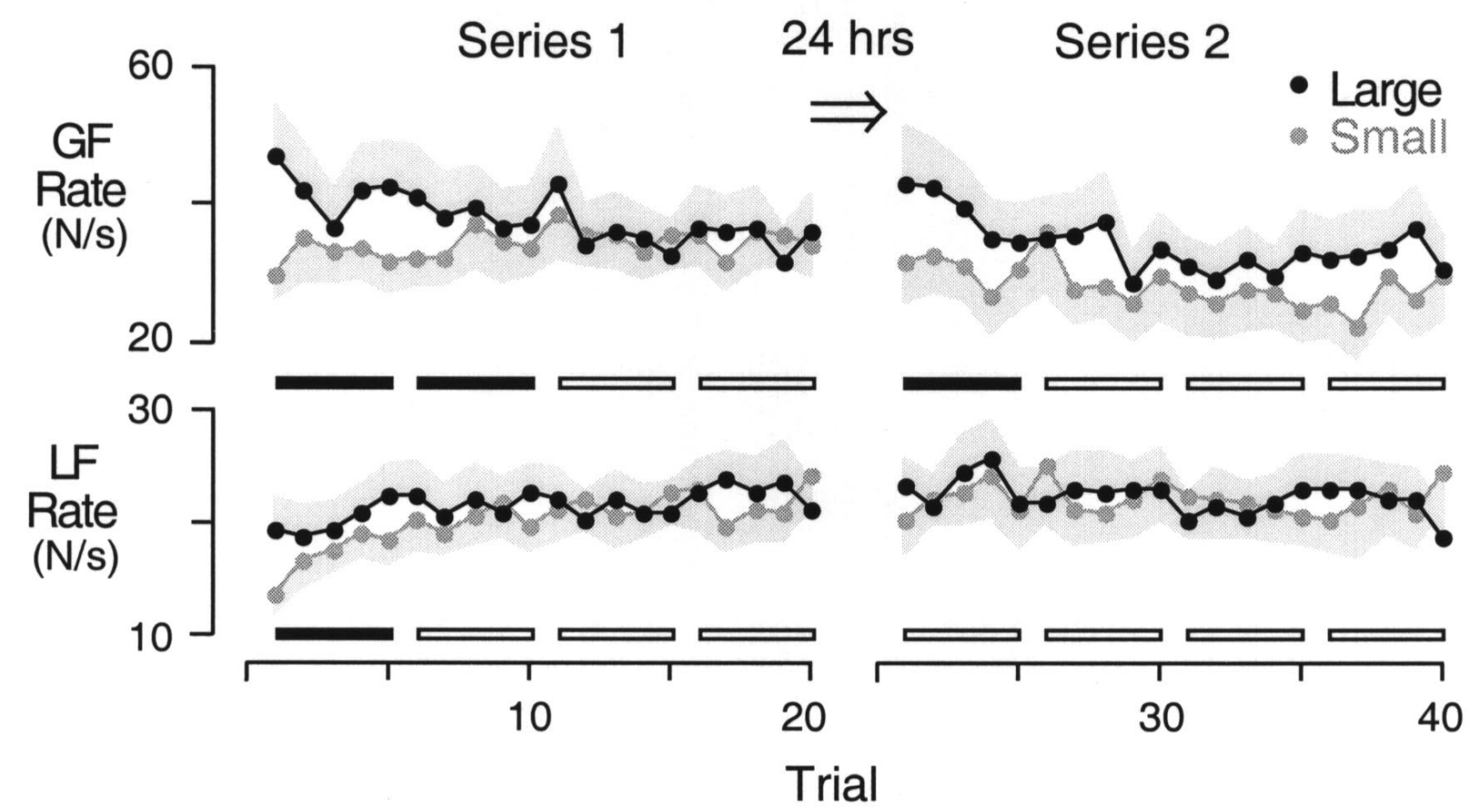

Figure 4. Recall after a 24- hour delay. Peak grip force rate (top) and peak load force rate (bottom) as a function of trial for two lift series performed a day apart. Each black (large cube) and gray (small cube) dot represents an average of nine participants. The gray regions represent one standard error. The horizontal bars indicate, for each block of five trials and for peak load force rate and peak grip force rate separately, whether there was (filled) or was not (open) a reliable effect of object size on the peak rate $(p<.05)$.

participants (Johansson \& Westling, 1984). Thus, the marked decline in this ratio in the initial few trials of the first lift series may arise because of changes in the actual frictional conditions or tuning of the safety margin to smaller values when the experimental situation becomes more familiar. Note that vision does not appear to be useful for predicting frictional conditions, probably because the friction between a given object and the fingertips may vary considerably depending on a number of factors related to skin lubrication such as sweating rate and grease (Johansson \& Westling, 1987). During the course of the lifting series, participants not only adapt to the true weight of the objects but also to the frictional conditions at the grasped surfaces.

In the first block of five trials of the second lift series (trials 21 to 25), neither peak grip force rate, $F(1,7)=.58, p=.47$ nor peak load force rate, $F(1,7)<$ $.01, p=.985$ were reliably affected by cube size. As shown in Figure 3, in the very first trial of the second lift series (trial 21), the mean peak force rates were greater for the large cube than the small cube. However, post-hoc examination of data from the first trial only failed to reveal a reliable effect of cube size on peak grip force rate, $F(1,8)=5.25, p>.01$ or peak load force rate, $F(1,8)=2.16, p>.01$. (With a Bonferroni correction, the required significance level reduces from .05 to .01 because there were five trials in the block and thus five possible comparisons.) Moreover, no significant interaction between cube size and trial was observed for either peak grip force rate, $F(4,28)=2.34, p=.079$ or peak load force rate, $F(4,28)$ $=1.56, p=.211$ in the first block of trials. Reliable effects of cube size on peak grip and load force rates were also absent in the second, third, and fourth blocks of the second lift series ( $p>.05$ in all six cases). These results demonstrate that participants retained sensorimotor memory related to lifting the cubes during the 15-minute delay period between the two lift series.

\section{SENSORIMOTOR MEMORY AFTER A 24-HOUR DELAY}

Figure 4 shows mean peak grip and load force rates for the large and small cubes as a function of trial. Data are shown for two lift series, each consisting of 20 trials, that were completed 24 hours apart. In the first block of five trials in the first lift series, greater peak grip, $F(1,12)=15.73, p=.002$ and load, $F(1,12)=5.61$, $p=.036$ force rates were observed during lifts of large cubes. In the second block of five trials, greater peak grip force rates, $F(1,12)=8.68, p=.012$ were observed when lifting the large cube but there was not a reliable effect of cube size on the peak load force rate, $F(1,12)$ $=2.55, p=.137$. In the third and fourth blocks of the 
first lift series, neither peak grip force rate nor peak load force rate was affected by cube size ( $p>.05$ in all four cases). Thus, as expected, fingertip forces gradually adapted over 5-10 trials such that they were similar for both cubes.

In the first block of five trials in the second lift series, greater peak grip force rates were observed when lifting the large cube, $F(1,14)=8.43, p=.012$. However, there was not a reliable effect of cube sized on the peak load force rate, $F(1,14)=1.78, p=.204$. In the subsequent three blocks of five trials in the second lift series, no significant effects of cube size on peak grip or load force rate were observed $(p>.05$ in all six cases).

The fact that load force rates were similar for the two cubes right from the start of the second lift series suggests that, even after a 24-hour delay, participants retained sensorimotor memory related to the weights of the two cubes. It should be stressed that load force rate provides a clearer index of expected weight than grip force rate because the latter is influenced by the frictional conditions at the grasped surfaces and the safety margin the participant employs. Participants may have employed greater safety margins when lifting the large cube in the second lift series because of greater uncertainty about the reliability of their predictions.

\section{Discussion}

In a previous study (Flanagan \& Beltzner, 2000), we demonstrated that when participants repeatedly lift large and small objects of equal weight in alternation, they learn to scale their fingertip forces so as to predict the true weights of the object within 5 to 10 lift pairs. This adaptation of forces is based on sensorimotor memory obtained during successive lifts and related to the forces required to lift the objects in a swift and smooth fashion. Participants learned to apply similar forces to the object despite the size difference by suppressing the use of visual size cues in force prediction. The aim of the present paper was to examine the stability of the sensorimotor memory mechanisms supporting this learning. First, we replicated our previous results demonstrating that sensorimotor memory wins out over visual size cues in terms of fingertip force prediction. We then showed that this sensorimotor memory is fully maintained for a period of 15 minutes and largely retained for 24 hours. This suggests that, in the first lift series, participants acquired lasting internal models of the two objects incorporating information about weight.

In the first lift series, fingertip forces were initially scaled, erroneously, to the visual size of the cubes. Thereafter, these forces were adjusted gradually to the true object weights over about five lifting trials. Thus, visual information about object size continues to influence predictive force control for a number of trials despite the fact that somatosensory information about the true object weights has been sampled in each lift (Westling \& Johansson, 1987). This gradual adaptation for fingertip forces is striking when contrasted with the one-trial learning observed when participants repeatedly lift a single object whose weight is erroneously predicted at first (Johansson \& Westling, 1988). In the Johannson and Westling (1988) study, the visual appearance of the object was constant and thus reliable information about weight changes was obtained by somatosensory mechanisms without conflicting visual information. Thus, one reason for the slow rate of force adaptation in the present experiments may be that participants had misleading visual size cues about weight. Another related reason for this slow rate of force adaptation may be that both cubes were of unusual density. Gordon and colleagues (1993) have shown that if participants repeatedly lift a single object with very high density $(4 \mathrm{~kg} / 1)$, the peak load force rate continues to increase over about five trials. These researchers also demonstrated that participants retain this adaptation when they return the next day and lift the same object. Our results extend this result by demonstrating that such retention is largely preserved even in the presence of potentially misleading visual size cues.

Visual size cues are effective if lifting a family of objects with common density. Given that the two objects used in the current experiments were similar in shape (i.e., they were both cubes), surface material, and colour, it seems reasonable to initially assume that they have the same density. Thus, in adapting fingertip forces to the true weights of the objects, participants had to learn that the two cubes had different density properties and thus belonged to different "families" in terms of size-weight correlation and thus size-force requirements.

In the 15-minute experiment, we observed that in the second lift series, participants correctly scaled their fingertip forces right from the very first trial. This indicates that they were able to use visual cues to select appropriate internal models from memory. In the second lift series of the 24-hour delay experiment, participants also correctly scaled load force right from the start. In contrast, grip force was initially scaled erroneously to the visual size of the objects. This may be due to greater uncertainly and/or caution when lifting the larger cube. Nevertheless, grip force was adapted more rapidly in the second lift series than in the first.

In general, both visual identification of object and sensory feedback obtained from lifting can be used effectively to select appropriate internal models stored in memory. How might this selection process work? 
One possible solution has been proposed by Wolpert and Kawato (1998) in the form of the modular selection and identification for control (MOSAIC) model (see Wolpert \& Ghahramani, 2000, for a review). The idea is that, when lifting an object, the brain simultaneously runs multiple forward models that mimic the behaviour of previously learned objects. Each forward model generates a prediction of the sensory feedback that should be obtained. Moreover, each forward model is paired with a corresponding inverse model forming a predictor-controller pair. If the prediction of one of the forward models closely matches the actual sensory feedback, then its paired inverse model will be selected and used to determine subsequent motor commands. (In computational terms, the sensory prediction error from a given forward model is represented as a probability; if the error is small then the probability that the forward model is appropriate is high. The set of probabilities from an array of forward models is used to weight the outputs of the corresponding inverse models.)

If multiple predictions are generated every time we lift an object, one may ask why, in the first lift series, fingertip forces were not adapted more rapidly. After all, most people will have experienced objects with unusually high or low density and would presumably have acquired internal models for these objects. In the MOSAIC model, the initial influence of a given forward model is determined by the context of the task. Thus, in the first lift series, the influence of (seldom used) forward models corresponding to objects of unusual density may be small. Consequently, despite the success of such forward models in predicting sensory outcomes, several trials may be required before their paired inverse models are fully selected. In contrast, in the second lift series, the recently used forward models for the size-weight stimuli may have a large influence right from the start. As a consequence, the paired inverse models will be rapidly selected.

In summary, we restate the main findings of this study. First, we replicated our previous study showing that sensorimotor memory comes to dominate visual size cues when lifting objects of equal weight but different volume. Participants learn to suppress visual size cues when predicting required forces. Second, we demonstrated that this sensorimotor memory is retained in memory and can be recalled a day later and possibly longer. This preserved sensorimotor memory may take the form of internal (forward and inverse) models that capture the mapping between motor commands and sensory outcomes.

Address correspondence to J. Randall Flanagan, Sara King, Department of Psychology and Canadian Institutes of Health Research Group in Sensory-Motor Systems, Queen's University,
Kingston, Ontario K7L 3N6; Daniel M. Wolpert, Sobell Department of Neurophysiology, Institute of Neurology, University College London, Queen Square, London WC1N 3BG, UK; Roland S. Johansson, Section for Physiology, Department of Integrative Medical Biology, Umeå University, SE-90187 Umeå, Sweden.

This work was supported by the Natural Sciences and Engineering Research Council of Canada, the Human Frontiers Science Program and the Wellcome Trust.

\section{References}

Charpentier, A. (1891). Analyse experimentale quelques elements de la sensation de poids [Experimental study of some aspects of weight perception]. Archives de Physiologie Normales et Pathologiques, 3, 122-135.

Davis, C. M., \& Roberts, W. (1976). Lifting movements in the size-weight illusion. Perception \& Psychophysics 20, 33-36.

Ellis, R. R., \& Lederman, S. J. (1998). The "golf-ball" illusion: Evidence for top-down processing in weight perception. Perception, 27, 193-202.

Flanagan, J. R., \& Beltzner, M. A. (2000). Independence of perceptual and sensorimotor predictions in the size-weight illusion. Nature Neuroscience, 3, 737-741.

Flanagan, J. R., \& Tresilian, J. R. (1994). Grip-load force coupling: A general control strategy for transporting objects. Journal of Experimental Psychology: Human Perception and Performance, 20, 944-957.

Flanagan, J. R., \& Wing, A. M. (1993). Modulation of grip force with load force during point-to-point arm movements. Experimental Brain Research, 95, 131-143.

Flanagan, J. R., \& Wing, A. M. (1995). The stability of precision grip force during cyclic arm movements with a hand-held load. Experimental Brain Research, 105, 455-464.

Flanagan, J. R., \& Wing, A. M. (1997). The role of internal models in motion planning and control: Evidence from grip force adjustments during movements of hand-held loads. Journal of Neuroscience, 17, 1519-1528.

Goodale, M. A., Meenan, J. P., Bülthoff, H. H., Nicolle, D. A., Murphy, K. J., \& Racicot, C. I. (1994). Separate visual pathways for the visual analysis of object shape in perception and prehension. Current Biology, 4, 604-610.

Goodale, M. A., Milner, A. D., Jakobson, L. S., \& Carey, D. P. (1991). A neurological dissociation between perceiving objects and grasping them. Nature, 349, 154-156.

Goodwin, A.W., Jenmalm, P., \& Johansson, R.S. (1998). Control of grip force when tilting objects: Effect of curvature of grasped surfaces and of applied tangential torque. Journal of Neuroscience, 18, 10724-10734.

Gordon, A. M., Forssberg, H., Johansson, R. S., \& Westling, G. (1991a). Visual size cues in the programming of manipulative forces during precision grip. Experimental Brain Research, 83, 477-482.

Gordon, A. M., Forssberg, H., Johansson, R. S., \& Westling, G. (1991b). The integration of haptically acquired size informa- 
tion in the programming of precision grip. Experimental Brain Research, 83, 483-488.

Gordon, A. M., Forssberg, H., Johansson, R. S., \& Westling, G. (1991c). The integration of sensory information during the programming of precision grip: Comments on the contribution of size cues. Experimental Brain Research, 85, 226-229.

Gordon, A. M., Westling, G., Cole, K. J., \& Johansson, R. S. (1993). Memory representations underlying motor commands used during manipulation of common and novel objects. Journal of Neurophysiology, 69, 1789-1796.

Granit, R. (1972). Constant errors in the execution and appreciation of movement. Brain, 95, 451-460.

Jenmalm, P., Dahlstedt, S., \& Johansson, R. S. (2000). Visual and tactile information about object curvature control fingertip forces and grasp kinematics in human dexterous manipulation. Journal of Neurophysiology, 84, 2984-2997.

Jenmalm, P., Goodwin, A. W., \& Johansson, R. S. (1998). Control of grasp stability when humans lift objects with different surface curvatures. Journal of Neurophysiology, 79, 1643-1652.

Jenmalm, P., \& Johansson, R. S. (1997). Visual and somatosensory information about object shape control manipulation fingertip forces. Journal of Neuroscience, 17, 4486-4499.

Johansson, R. S. (1998). Sensory input and control of grip. In M. Glickstein (Ed.), Sensory Guidance of Movement (pp. 45-59). Chichster, uK: John Wiley.

Johansson, R. S., Backlin, J. L., \& Burstedt, M. K. O. (1999). Control of grasp stability during pronation and supination movements. Experimental Brain Research, 128, 20-30.

Johansson, R. S., \& Cole, K. J. (1994). Grasp stability during manipulative actions. Canadian Journal of Physiology and Pharmacology, 72, 511-524.

Johansson, R. S., \& Westling, G. (1984). Roles of glabrous skin receptors and sensorimotor memory in automatic control of precision grip when lifting rougher or more slippery objects. Experimental Brain Research, 56, 550-564.

Johansson, R. S., \& Westling, G. (1987). Signals in tactile afferents from the fingers eliciting adaptive motor responses during precision grip. Experimental Brain Research, 66, 141-154.

Johansson, R. S., \& Westling, G. (1988). Coordinated isometric muscle commands adequately and erroneously programmed for the weight during lifting task with precision grip. Experimental Brain Research, 71, 59-71.
Jordan, M.I., \& Rumelhart, D.E. (1992). Forward models: supervised learning with a distal teacher. Cognitive Science, 16, 307-354.

Kawato, M. (1999). Internal models for motor control and trajectory planning. Current Opinions in Neurobiology, 9, 718-727.

Kawato, M., Furukawa, K., \& Suzuki, R. (1987). A hierarchical neural network model for the control and learning of voluntary movements. Biological Cybernetics, 56, 1-17.

Kinoshita, H., Bäckström, L., Flanagan, J. R., \& Johansson, R. S. (1997). Planar torque effects on grip force during precision grip. Journal of Neurophysiology, 78, 1619-1630.

Miall, R. C., \& Wolpert, D. M. (1996). Forward models for physiological motor control. Neural Networks, 9, 1265-1279.

Milner, A. D., \& Goodale, M. A. (1995) The visual brain in action. Oxford UK; Oxford University Press.

Murray, D. J., Ellis, R. R., Bandomir, C. A., \& Ross, H. E. (1999). Charpentier (1891) on the Size-weight Illusion. Perception $\&$ Psychophysics, 61, 1681-1685.

Ross, H. E. (1969). When is a weight not illusory? Quarterly Journal of Experimental Psychology, 21, 346-355.

Sperry, R. W. (1950). Neural basis of spontaneous optokinetic responses produced by visual inversion. Journal of Comparitive Physiology and Psychology, 43, 482-489.

Von Holst, E. (1954). Relations between the central nervous system and the peripheral organs. British Journal of Animal Behaviour, 2, 89-94.

Westling, G., \& Johansson, R. S. (1987). Responses in glabrous skin mechanoreceptors during precision grip in humans. Experimental Brain Research, 66, 128-140.

Wing, A. M., \& Lederman, S. J. (1998). Anticipating load torques produced by voluntary movements. Journal of Experimental Psychology: Human Perception and Performance, 24, 1571-1581.

Wolpert, D. M., \& Ghahramani, Z. (2000). Computational principles of movement neuroscience. Nature Neuroscience, 3, 1212-1217.

Wolpert, D. M., Ghahramani, Z., \& Jordan, M. I. (1995). An internal model for sensorimotor integration. Science, 269, 1880-1882.

Wolpert, D. M., \& Kawato, M. (1998). Multiple paired forward and inverse models for motor control. Neural Networks, 11, 1317-1329. 